pharmacy dispensing data for $80 \%$ of the population in Catalonia ( 6 million people). All persons aged 18 or older at the beginning of each calendar year with an incident $O A$ diagnosis (including both peripheral and central joints) in the study period were included. Index date was the date of first OA diagnosis, and the observation period of opioid use was 1-year after index date. Opioids considered included codeine, tramadol, fentanyl, and morphine, with the latter three classified as strong opioids. The period prevalence of any opioid use was estimated in whole and sub-population stratified by sex, age, socio-economic status (U1 - U5, higher values of the indicator equivalent to deprivation) and residence area (rural/urban).

Results: The 1-year prevalence of any opioid use among incident OA patients was around $15 \%$ from 2007 to 2012 . After that, this figure grew by $10 \%$ approaching $25 \%$ in 2016 . However, strong opioid use increased continuously to nearly triple, from $8 \%$ in 2007 to $20 \%$ in 2016 . The different subgroups followed similar trends over time, with women $4 \%$ higher than men, oldest $10 \%$ higher than youngest, most deprived $6 \%$ higher than least deprived, and rural $1 \%$ higher than urban.

Conclusion: The use of opioids (and especially strong opioids) has substantially increased in recent years among newly diagnosed OA patients in Catalonia. Our findings call for urgent action for safe opioid prescribing to avoid opioid abuse in OA patients especially amongst older women living in deprived areas.

Disclosure of Interests: Junqing Xie: None declared, Aleksandra Turkiewicz: None declared, Gary Collins: None declared, Martin Englund Consultant of: Advisory Board 1 day (2019) Pfizer (Tanezumab)., Victoria Y Strauss: None declared, Carlen Reyes: None declared, Daniel Prieto-Alhambra Grant/research support from: Professor Prieto-Alhambra has received research Grants from AMGEN, UCB Biopharma and Les Laboratoires Servier, Consultant of: DPA's department has received fees for consultancy services from UCB Biopharma, Speakers bureau: DPA's department has received fees for speaker and advisory board membership services from Amgen

DOI: 10.1136/annrheumdis-2020-eular.3070

\section{OP0281 TWO-YEAR COST-EFFECTIVENESS BETWEEN TWO GRADUAL TAPERING STRATEGIES IN RHEUMATOID ARTHRITIS: COST-UTILITY ANALYSIS OF THE TARA} TRIAL

E. Van Mulligen ${ }^{1}$, A. Weel ${ }^{2,3}$, M. Kuijper ${ }^{2}$, J. Hazes ${ }^{1}$, P. De Jong ${ }^{1} .{ }^{1}$ Erasmus $^{2}$ MC, Rotterdam, Netherlands; ${ }^{2}$ Maasstad Hospital, Rotterdam, Netherlands; ${ }^{3}$ Erasmus School of Health Policy \& Management, Rotterdam, Netherlands

Background: Current guidelines recommend to consider tapering treatment in rheumatoid arthritis (RA) patients who are in sustained remission, but the optimal approach to de-escalate conventional synthetic and biological DMARDs (respectively csDMARDs and bDMARDs) remains unknown. The benefits of tapering are a decreased risk of long-term adverse events and a reduction of health care costs, especially when bDMARDs are tapered. However, tapering treatment may lead to more transient or persistent disease flares, which have a direct impact on patients' lives and societal costs.

Objectives: To evaluate the two year cost-utility ratio between tapering the csDMARD first followed by the TNF-inhibitor, and tapering the TNF-inhibitor first followed by the cSDMARD.

Methods: The TARA trial is a multicenter single-blinded randomized controlled trial. RA patients that used a csDMARD(s) plus a TNF-inhibitor and who had a well-controlled disease for at least 3 months, defined as a DAS $\leq 2.4$ and a swollen joint count $(\mathrm{SJC}) \leq 1$, were included. Patients were randomized into gradual tapering their csDMARD followed by the TNF-inhibitor or vice versa. Medication was tapered in three steps over the course of 6 months. Gradual tapering was done by cutting the dosage into half, a quarter and thereafter it was stopped. Data on QALYS (measured with the Dutch EuroQol [EQ5D]), direct and indirect costs were used to calculate the Incremental Cost Effectiveness Ratio (ICER). The incremental cost-effectiveness ratio (ICER) and the incremental net monetary benefit (iNMB) were used to assess cost-effectiveness between both tapering strategies. Direct costs comprises costs for treatment and medical consumption, while indirect costs comprises costs due to loss of productivity (i.e. sick leave and unemployment).

Results: Of the 189 included patients, 94 started tapering their TNF-inhibitor first, while the other 95 tapered their csDMARD first. QALYs (sd) were, respectively, $1.64(0.22)$ and $1.65(0.22)$. Medication costs were significantly lower in the patients who tapered the TNF-inhibitor first, but indirect cost were higher due to more productivity loss. Therefore, total costs per QALY were similar for both tapering strategies $(p=0.62)$. The ICER between tapering csDMARDs and the TNF-inhibitor was $-€ 184534$ (-€417314, €48245; 95\% Cl)(figure 1). The mean iNMB was $€ 2831$ at a willingness-to-pay (WTP) level of $€ 80000$. At all WTP levels the probability of being cost-effective was higher (62\% vs. $28 \%$ ) for tapering the TNF-inhibitor first (figure 2)

Conclusion: Medication costs are lower when the TNF-inhibitor is tapered first, but this is counterbalanced by higher indirect costs due to loss of productivity.
Therefore, overall cost savings are smilar for both tapering strategies. However, tapering the TNF-inhibitor first has a higher chance of being cost-effective at all WTP thresholds. For this reason we advise to taper the TNF-inhibitor first when tapering medication is considered.

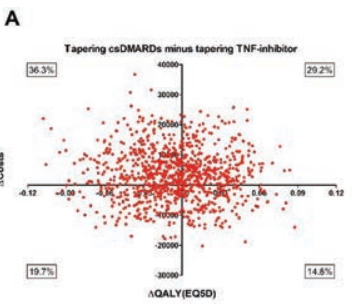

B

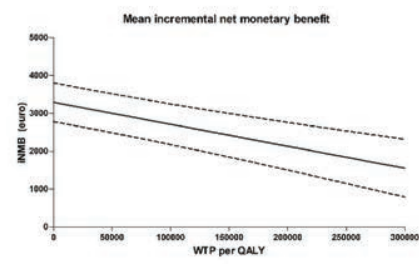
Figure 1 Summary of economic evaluation of tapering csDMARDs first minus tapering INF-inhibitor first. (A)
Results of 1000 bootstrapped replications, presented in a cost-effectiveness plane which represents uncertainty of the
cost-effectiveness ratio. (B) Mean incremental net monetary beneffit for tapering cSDMARDs minus tapering TNF-inhibitors cost-effectiveness ratio. (B) Mean incremental net monetary benefit for tapering csDMARDS minus tapering TNF-inhibitors
with 5 \% c confidence intervals slotted against different tevels of willingness to pay (WTP) per quality adjusted life year (QALY). CsDMARDs: conven:
year; WTP: willingness to pay

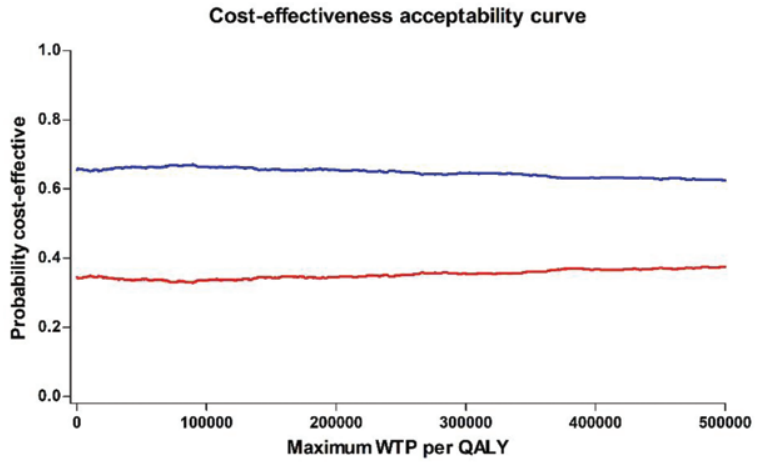

Tapering TNF-inhibitor first Tapering csDMARDs first

Figure 2 Cost effectiveness acceptability curve for tapering csDMARDs first versus tapering TNF-inhibitor first. Results of 1000 bootstrapped replication, presented for several levels of willingness to pay, indicated per quality adjusted life year (QALY). csDMARDs: conventional synthetic DMARDs; QALY: quality adjusted life year; WTP: willingness to pay.

Disclosure of Interests: None declared DOI: 10.1136/annrheumdis-2020-eular.740

\section{OP0282 1 COST-EFFECTIVENESS ANALYSIS OF A CAFASPA REFERRAL MODEL FOR AXIAL SPONDYLOARTHRITIS}

M. J. Abdelkadir ${ }^{1}$, M. Kuijper ${ }^{1}$, C. Appels ${ }^{2}$, A. Spoorenberg ${ }^{3}$, J. Hazes ${ }^{4}$, L. Van Hoeven $^{4}$, D. Lopes Barreto ${ }^{1}$, A. Weel ${ }^{1,5}$. 'Maasstad Ziekenhuis, Rheumatology, Rotterdam, Netherlands; ${ }^{2}$ Amphia Hospital, Breda, Netherlands; ${ }^{3}$ University Medical Center Groningen, Groningen, Netherlands; ${ }^{4}$ Erasmus Medical Center, Rotterdam, Netherlands; ${ }^{5}$ Erasmus School Health Policy \& Management, Rotterdam, Netherlands

Background: Chronic low back pain (CLBP) poses a significant individual and socio-economic burden. A substantial amount of patients with CLBP have axia spondyloarthritis (axSpA), but early recognition of these patients is difficult for general practitioners (GPs). Guidelines form primary care and secondary care differ in criteria for referral recommendation. The Dutch primary care guideline is restrictive in referring CLBP patients to secondary care whereas ASAS recommend to refer CLBP patients having at least 1 axSPA feature ${ }^{1}$. Therefore several referral models have been developed to assist GPs. Although the validated CaFaSpA referral model ${ }^{2}$ is able to identify CLBP patients at risk for axSpA, its cost-effectiveness is yet unknown and essential before implementation in daily clinical practice.

Objectives: Primary objective to assess the cost-effectiveness of the CaFaSpA referral model for axSpA in primary care. Secondary objective to evaluate the costs made for screening by following the CaFaSpA vs ASAS referral model.

Methods: A clustered randomized controlled trial was performed with GPs as clusters. Clusters were randomized into the intervention (CaFaSpA referral, CS) or usual care (UC). Cost-effectiveness analysis from a societal perspective was performed to compare the CS and UC. Clinical outcomes were disability 